\title{
Acupuncture Treatments for Post-stroke Depression: A Systematic Review and Network Meta-analysis
}

\author{
Lam Wai Ching \\ Hong Kong Baptist University School of Chinese Medicine \\ Hui Juan Li \\ Hong Kong Baptist University School of Chinese Medicine \\ Jianwen Guo \\ Guangdong Medical University \\ Liang Yao \\ McMaster University \\ Janita Chau \\ The Chinese University of Hong Kong \\ Suzanne Lo \\ The Chinese University of Hong Kong \\ Chun Sum Yuen \\ Hong Kong Baptist University \\ Bacon Fung Leung $\mathrm{Ng}$ \\ PolyU: The Hong Kong Polytechnic University \\ Edwin Chau-Leung Yu \\ Hong Kong Baptist College: Hong Kong Baptist University \\ Zhaoxiang Bian \\ Hong Kong Baptist University \\ Alexander $Y$ Lau \\ Prince of Wales Hospital \\ Linda LD Zhong ( $\square$ ldzhong@hkbu.edu.hk) \\ Hong Kong Baptist University
}

\section{Research}

Keywords: Acupuncture, Post-stroke depression, Network meta-analysis

Posted Date: October 12th, 2021

DOI: https://doi.org/10.21203/rs.3.rs-951353/v1

License: (c) (i) This work is licensed under a Creative Commons Attribution 4.0 International License. Read Full License 


\section{Abstract}

Background: Depression is one of the most common complications after stroke, with a prevalence of $30-33 \%$. Patients with post-stroke depression (PSD) usually experience anxiety, hopelessness, and insomnia, which have a negative impact on their daily activities and post-stroke rehabilitation.

In this review, we aimed to explore the impact of acupuncture in alleviating symptoms of PSD and to evaluate the difference in effectiveness between acupuncture combined with pharmacotherapies and various non-pharmacotherapies in order to provide guides and advice for clinical personnel.

Methods: Six databases (Cochrane Library, PubMed, EMBASE, China National Knowledge Infrastructure, Wanfang Database, Chongqing VIP Database) and two clinical trials registration platforms were searched from inception to May 2021. Randomized clinical trials (RCTs) comparing needle-based acupuncture with pharmacotherapy, and other non-pharmacotherapy or invalid group were included. Two independent reviewers identified eligible studies. Two reviewers independently abstracted and recorded data into a pre-made form. A Bayesian network meta-analysis (NMA) was conducted to assess and compare different techniques using RStudio 3.6.0. The primary outcome was the change in scores of scales measuring depressive symptoms. The secondary outcomes were changes in serotonin levels and in quality of life.

Results: Fifty-nine RCTs were included. The results of this NMA showed that compared with western medicine (WM), acupuncture alone or with repetitive transcranial magnetic stimulation (RTMS), Traditional Chinese medicine (TCM) alone or with WM, were superior for alleviating depression symptoms. Compared to Usual Care, acupuncture alone or plus other therapies could significantly decrease scores of the Hamilton Depression Rating scale. However, there was no significant difference found among acupuncture, WM, TCM with AC plus any of other treatment.

Conclusions: The results of this study indicate that acupuncture alone or combined with other therapies appear to be effective in improving depression symptoms of stroke survivors. Moreover, in comparison with WM, acupuncture alone or plus RTMS, TCM, TCM with WM, or WM, were more effective in improving depression symptoms. Also, acupuncture with RTMS seems to be the most effective with the highest probability.

\section{Background}

Stroke is the second leading cause of death according to the World Health Organization (WHO) in 2019, responsible for approximately $11 \%$ of total deaths. ${ }^{1}$ Moreover, it is also one of the leading causes of disability worldwide, and causes the loss of a great number of healthy life-years due to serious post-stroke sequelae (such as paralysis, aphasia, dysphagia, epilepsy, and cognitive difficulty). ${ }^{2-5}$ Facing severe symptoms and different physical disabilities, stroke survivors might face tremendous health challenges, and they are more likely to suffer from psychological disorders. Depression is one of the most common complications after stroke, with a prevalence of $30-33 \%{ }^{6-9}$ Patients with post-stroke depression (PSD) usually experience anxiety, hopelessness, unwillingness to communicate, and insomnia, which could have a negative impact on the daily activities and post-stroke rehabilitation. ${ }^{10}$ Moreover, it is suggested that PSD is associated with an increased risk of mortality in stroke survivors. ${ }^{6}$ Therefore, it is quite crucial to devise an effective and safe treatment for PSD. The selective serotonin reuptake inhibitors (SSRIs) are commonly recommended as the first-line pharmacological treatments. ${ }^{11}$ However, there still exists a debate on whether SSRIs could increase the risk of intracerebral hemorrhage and subsequent stroke. ${ }^{12,13}$

Acupuncture, as a minimal invasive technique, has been widely used for improving symptoms of a variety of health problems in China and worldwide. ${ }^{14-16}$ Many published studies investigated the effectiveness of acupuncture for PSD. One meta-analysis by Xin Yan Zhang etc. included seven studies comparing the effectiveness rate of acupuncture with control group in alleviating the symptoms of PSD. Their results supported that acupuncture was an effective and safe treatment for PSD ${ }^{17}$ (RR 1.16, 95\% Cl 1.08-1.24). Another study by Xue Wang etc. evaluated the effectiveness of acupuncture combined with western medicine for PSD based on 24 studies. The meta-analysis showed acupuncture combined with fluoxetine was superior to fluoxetine alone for relieving depressive symptoms. ${ }^{18}$ Moreover, more clinical trails evaluated whether there was a better effect when AC combined other non-pharmacotherapies for PSD. The study by Zhang LIN etc. investigated the efficacy of AC plus Tai Chi in recovering the neurological function and treating depression in PSD. ${ }^{19}$ Another study compared the difference of $\mathrm{AC}$ combined plus cognitive therapy with paroxetine. ${ }^{20}$ The authors of Yaqun WANG etc. enrolled 103 participants to evaluate the effect of AC plus Jieyu Qingxin Decotion (a Traditional Chinese herbal medicine) combined compared with paroxetine.

In this review, we seek to explore the true effect of acupuncture in alleviating symptoms of PSD based on both direct and indirect evidence using the network meta-analysis (NMA) method. ${ }^{21}$ Moreover, we also aim to evaluate the difference in effectiveness between acupuncture with pharmacotherapies and other non-pharmacotherapies in order to provide optimized guidance and advice for clinical personnel.

\section{Methods}


This review was reported in accordance with the Preferred Reporting Items for Systematic Review and Meta-Analyses (PRISMA) for Network Meta-Analyses, ${ }^{22}$ and was registered in the International Prospective Register of Systematic Reviews (PROSPERO) database in November 2020 and updated in July 2021 (Registration no.: CRD42020218752) 23.

\section{Literature search}

The Cochrane Library, PubMed, EMBASE, China National Knowledge Infrastructure (CNKI), Wanfang Database, Chongqing VIP Database (CQVIP) were searched for systematic reviews of published articles from inception to May 2021. The clinical trials registration platform and websites were additionally searched, including the International Clinical Trials Registry Platform (ICTRP), ClinicalTrials.gov, ISRCTN registry, https://scholar.google.com.tw/, https://xueshu.baidu.com/. There was no restriction in language or publication year. Backward and forward reference searching was applied. The references of relevant reviews and systematic reviews were retrieved to search for potential eligible studies. The search strategy of this study is shown in Supplementary Appendix 1.

\section{Inclusion Criteria}

Studies comparing needle-based acupuncture (alone or combined with other treatments) with pharmacotherapy, other non-pharmacotherapy or invalid group (including placebo, waitlist and blank control) were included.

P - Participants who were clinically diagnosed with stroke, 18 years or older, and with any degree of stroke impairment severity and at any stage of the disease.

I - Acupuncture treatments were specified to needle-based acupuncture, including but not limited to manual, electro-acupuncture, fire acupuncture, warm acupuncture, ear (auricular) acupuncture, head acupuncture, and more.

C - Acupuncture treatments alone or combined with other treatments (pharmacotherapy and non- pharmacotherapy therapies) were compared with pharmacotherapy, other non-pharmacotherapy or invalid groups (including placebo, waitlist and blank control).

0 - Outcomes reflecting changes in scores of PSD.

S - Only randomized controlled trial (RCT).

Studies comparingwith different types of acupuncture were excluded.

\section{Literature Selection and Data Extraction}

Two independent reviewers extracted data from selected RCTs. The basic characteristics such as first author, study title, participants (gender, age, sample size), study design (randomization, blinding), details of interventions, outcome measures, results and adverse events were abstracted and recorded into a pre-made form. Any disagreements were reviewed by a third reviewer and resolved by discussion among all reviewers.

\section{Risk of Bias Assessment}

The risks of bias of included RCTs were assessed based on the Revised Cochrane Risk of Bias tool $2 .{ }^{24}$ which five domains were evaluated. The appraisal of acupuncture procedures was assessed by Revised Standards for Reporting Interventions in Clinical Trials of Acupuncture (STRICTA). ${ }^{25}$ The Grading of Recommendations Assessment, Development and Evaluation (GRADE) was used to assess the certainty of the evidence. ${ }^{26,27}$

\section{Statistical Analysis \\ Pairwise meta-analysis}

Risk ratios with 95\% credible intervals were used for dichotomous outcomes. Mean differences or standardized mean differences with $95 \%$ credible intervals were used for continuous outcomes. We assessed clinical and methodological heterogeneity through examination of the characteristics of the included trials. Heterogeneity across trials was assessed by $X^{2}$ and $l^{2}$ statistics. Publication bias was examined using Begg's and Egger's funnel plot method when applicable. The contour-enhanced funnel plot was obtained as an aid to distinguish asymmetry due to publication bias.

Network meta-analysis 
A network plot was drawn to present the geometry of the network of comparisons across trials to ensure the network meta-analyses was feasible. Trials were excluded if they were not connected by interventions.

We performed Bayesian network meta-analyses to compare the effects of different prophylactic agents. Markov chains were used to generate samples. Model convergence was assessed using the Brooks-Gelman-Rubin plots method. Global heterogeneity was assessed on the bias of the magnitude of heterogeneity variance parameter estimated from the network meta-analyses models. A node-splitting method was used to examine the inconsistency between direct and indirect comparisons when a loop connecting three arms existed. The ranking probabilities for all treatments were estimated, and a treatment hierarchy using the probability of being the best treatment could be obtained. This process was performed using the Surface Under the Cumulative Ranking curve (SUCRA). We used the frequentist approach to compare stability if necessary. Statistical analysis was performed with STATA 15.0 and RStudio 3.6.0.

\section{Results}

\section{Search Results}

Initially, 7,472 records were identified. After screening titles and abstracts, 7,406 records were excluded and the remaining were considered potentially eligible for full-text screening. Finally, 59 studies $20,28-86$ involving 5308 participants published from 2003 to 2021 were included in this review. The flow chart of process of the study selection and studies considered for inclusion is shown in Supplementary Appendix 2.

\section{Characteristics of Included Studies}

The main characteristics of the included studies are shown in Table 1. Fifty-nine studies involving 5,308 participants were included. All of them were from China. Apart from Usual Care (UC) (defined as controlling blood pressure and blood sugar, physical exercises or pharmacotherapy for stroke rehabilitation, and no special treatment for depression), twelve various treatments were included. Among them, acupuncture (AC) with pharmacotherapy, which is divided into AC with Western Medicine (WM) and AC with Traditional Chinese Medicine (TCM), (in this case, traditional Chinese herbal remedies), and AC with both TCM and WM. There were also five therapies comprising AC plus non-pharmacotherapy, including repetitive transcranial magnetic stimulation (RTMS), Tai Chi, Cognitive Therapy (CT), psychotherapy, and moxibustion (AM). There was one RCT exploring the treatment difference between AC with RTMS and AC alone. One study investigated the efficacy of AC combined with Tai Chi in comparison with WM. Another study evaluated AC combined with psychotherapy. Eleven studies were divided into three groups, while others were based on two groups. Twenty-eight explored the effect of AC alone in comparison with WM or UC.

\section{Assessment Results of Risk of Bias and Reporting Quality Based on STRICTA}

The quality assessment results are given in Supplementary Appendix 3. Seven studies $(7,11.86 \%)$ had low risk of bias arising from the randomization process (Domain 1). Nine studies $(9,15.25 \%)$ were evaluated as "low" for the risk of bias due to deviations from the intended interventions (Domain 2). In terms of overall risk of bias, 26 studies were ranked as "high", 27 as "some concern”, and 6 as "low”.

The assessment results of reporting quality showed that all of the studies (100\%) reported the style of acupuncture (Item $1 \mathrm{a})$, and $38 \mathrm{RCTS}$ (64.4\%) demonstrated the needle type (Item 2g). There were 39 (66.1\%) studies that presented the "response sought" (Item $2 \mathrm{~d}$ ). Only 18 (30.5\%) reported the "number of needle insertions per subject per session" (Item 1c). None illustrated the "setting and context of treatment, including instructions to practitioners, and information and explanations to patients" (Item 4b) and "rationale for the control or comparator" (Item 6a). The details of the STRICTA assessment for each study are listed in Supplementary Appendix 4.

\section{Network meta-analysis}

The network evidence plots were presented in Figure 1. The main results of the NMA for depression. Fifty studies involving 4,547 patients reported changes in depression scores in the HAMD scale, 21 for HAMD-17, 1 for HAMD-21, 28 for HAMD-24. The scores of different versions of HAMD were standardized. One study comparing the treatment of acupuncture combined with psychotherapy was excluded due to its failure to directly or indirectly connect with other interventions. ${ }^{78}$ Six three-armed-based studies and 43 two-arm based studies were included. A total of 104 arms were included, 41 arms for WM, 28 for AC, 11 for AC with WM, 7 for UC, 4 for AC with TCM, 4 for AM with WM, 2 for AM with TCM with WM, 2 for TCM, 1 arm for AC with RTMS, 1 for AC with Tai Chi, 1 arm for AM with TCM, 1 for AC with CT, 1 for AM. Among these studies, the largest number of studies were those comparing AC with WM $(n=20)$. The following comparison type of studies were AC with WM versus WM $(11,22 \%)$ and $A C$ versus UC $(7,14 \%)$. The main results of the NMA for depression are displayed in Table 2.

On the whole, interventions administering combined therapies were more effective in comparison with those using single therapy. The results of NMA showed that compared with WM alone, the administration of AC with RTMS, AC with TCM and WM, AC with TCM, TCM alone, AC with WM, and $\mathrm{AC}$ alone were superior in alleviating depression symptoms (MD, -8.75 95\% Cl: -16.75, -0.76; $\mathrm{MD},-5.83,95 \% \mathrm{Cl}$ : $-11.22,-0.42 ; \mathrm{MD},-6.68,95 \%$ Cl: -10.16, -3.18; MD, -5.43, 95\% Cl: -10.73, -0.13; MD, -4.34, 95\% Cl: -6.64, -2.05; MD, -3.49, 95\% Cl: -5.19, -1.79, respectively). Compared to UC, AC 
alone or in combination with other interventions could lower HAMD scores (AC with RTMS. MD, -14.43, 95\% Cl: -22.81, -6.08; AC with TCM with WM: MD, -11.51, 95\% Cl: -17.85, -5.17; AC with TCM: MD, -12.35, 95\% Cl: -17.11, -7.62; AC with Tai Chi: MD, -10.19, 95\% Cl: -18.59, -1.83; AC with CT: MD, -9.64, 95\% Cl: -18.10, -1.21; TCM: MD, -11.11, 95\% Cl: -17.28, -4.99; AM: MD, -8.02, 95\% Cl: -15.73, -0.34; AC with WM: MD, -10.02, 95\% Cl: -14.04, -6.04; AM with WM: MD, -8.03, 95\% Cl: -13.17, -2.94; AC. MD, -9.17, 95\% Cl: -12.17, -6.22; WM: MD, -5.68, 95\% Cl: -9.04, -2.33). However, no significant difference was found among AC, WM, and TCM with AC plus any other treatment (AC with RTMS, AC with TCM, AC with TCM with WM, AC with Tai Chi, AC with WM, AC with CT, AM and AM with WM).

Examination of consistency with the node-splitting analysis approach indicated that there was no significant inconsistency $(P>0.05)$ (Supplementary Appendix 5). The analysis of heterogeneity showed the $R^{2}$ of direct comparison was $97.66 \%$, and the global $R^{2}$ was $97.38 \%$. The $R^{2}$ of each comparison group was seen in Supplementary Appendix 6.

Table 3 presented the values of SUCRA, the hierarchy of thirteen treatments. According to SUCRA, AC plus RTMS had the highest probability in improving depressive symptoms with probability of 49.43\%. The next were AC with TCM with WM (10.99\%), AC with TCM (10.62\%), AC with Tai Chi (10.30\%), which were very close. The probability of AC with CT and TCM were $8.10 \%$ and $7.48 \%$ respectively. The figure of SUCRA was attached in Supplementary Appendix 7.

\section{Pairwise Meta-Analysis}

Ten studies $56,58,64$ 40,45,54,55,62 29,36 used the Modified Edinburgh-Scandinavian Stroke Scale (MESSS) to measure neurological impairment. The of result of pairwise meta-analysis showed that AC was significantly associated with better neurological function improvement than UC or WM (MD, -8.45, 95\% Cl: $-12.85,-4.05$; MD, $-5.11,95 \%$ Cl: $-6.50,-3.73$, respectively). Similarly, AC with WM and AC with TCM was superior to WM (MD, $-5.07,95 \% \mathrm{Cl}:-8.41,-1.73$; MD, $-5.32,95 \% \mathrm{Cl}:-8.89,-1.66$, respectively) (Supplementary Appendix 8).

\section{The confidence assessment result of NMA}

The CINeMA system (https://cinema.ispm.unibe.ch/) was used to classify the confidence in the results of NMA, which six domains was evaluated, including: (a) within- study bias, (b) reporting bias, (c) indirectness, (d) imprecision, (e) heterogeneity, and (f) incoherence. One comparison (AC versus AC with TCM) was ranked "high"; eight were "moderate"; eight were "low". The details are presented in Table 4.

\section{Discussion}

This network meta-analysis found that compared with WM, AC alone or in combination with RTMS, TCM, WM, or TCM with WM seemed to be more effective in improving depression symptoms. When compared with UC, AC alone or combined with any other therapy (including AC with RTMS, AC with TCM, AC with TCM and WM, AC with Tai Chi, AC with WM, AC with CT, AM, and AM with WM) was superior. However, we did not find any significant difference between any comparisons of these interventions. Additionally, the pairwise meta-analysis showed that combined therapies of $\mathrm{AC}$ with WM, AC with TCM, and AC alone were significantly associated with better neurological function improvement in contrast to UC or WM.

The result of SUCRA showed that AC with RTMS had the highest probability of being the most effective therapy to alleviate depressive symptoms. It is reported that RTMS, as a non-invasive approach, could induce excitability changes in the motor cortex via a wire coil generating a magnetic field that passes through the scalp. However, the physiological mechanism of RTMS after-effects is yet to be clear. ${ }^{89}$ The updated evidence-based guidelines by Lefaucheur et al. demonstrated that RTMS has a significant effect in several psychiatric diseases. It is also highly recommended (Level A) for major depression, ${ }^{90}$ which was consistent with our findings. Nonetheless, it needs to be interpreted with caution because there was only one eligible study included which compared AC alone and AC with RTMS. More relevant research is needed to confirm the true function and safety of RTMS for patients with PSD.

In this study, various combined therapies were evaluated, including AC with RTMS, AM with TCM, AC with Tai Chi, AC with TCM with WM, AC with TCM, AC with CT, AC with WM, AM with WM. We first explored the difference in effectiveness for PSD between these therapies using the network meta-analysis approach, which synthesized the direct comparison evidence and indirect comparison evidence. However, some comparisons contained only a few eligible studies, such as those on AC with RTMS versus AC, AC with CT versus WM, and AC with Tai Chi versus WM. Therefore, it is suggested that future research could focus on the effectiveness of these different comparisons for patients with PSD. We will also conduct an update if further studies are published.

One published network meta-analysis by Hang et al. compared the effect of different acupuncture approaches for specific parts in treating patients with PSD. ${ }^{91}$ Twelve acupuncture therapies were included in their study, and they found that scalp acupuncture plus conventional acupuncture was the most effective method based on the ranking probability. For this study, we put more focus on the effectiveness of acupuncture combined with other therapies for PSD. Due to the complexity in the nature of acupuncture treatments and their procedure, we were not able to conduct a relevant subgroup analysis. Therefore, we could only differentiate the types of acupuncture by their main characteristics,

Page 5/16 
which induced huge heterogeneity among acupuncture treatments. The details were presented in Supplementary Appendix 9 . The most frequently used acupoints included Bai-Hui, Shen-Ting, and Nei-Guan.

This is the first review to compare the effectiveness of acupuncture with other therapies for PSD using network meta-analysis, which may provide novel and useful guidance for clinicians and readers. However, there are still several limitations. Firstly, although we conducted a systematic search for eligible studies, only Chinese studies were eligible and subsequently included. Secondly, the newest assessment tool of ROB 2 was used to evaluate the quality of included RCTs, and the assessment result indicated that the overall quality of RCTs was not high. Therefore, more high-quality RCTs are needed.

\section{Conclusion}

In our review, we provide an overview of the current research evidence on the effectiveness of AC alone or in combination with other therapies for the treatment of PSD. Twelve various treatments are included. Among them, there are five therapies of AC plus non-pharmacotherapy (RTMS, Tai Chi, CT, psychotherapy, moxibustion), while AC plus pharmacotherapy treatments also include AC with WM and AC with TCM. The results of NMA indicate that combined therapies, including AC with RTMS, AC with TCM, AC with TCM with WM, AC with WM, and AC alone may be more effective in alleviating depression symptoms as compared with WM. With the highest probability, AC with RTMS seems to be the most effective with the highest probability in treating PSD. Nonetheless, more high-quality studies are needed to provide sufficient evidence.

\section{Declarations}

\section{Acknowledgements}

Not applicable.

\section{Ethics approval and consent to participate}

Findings from the study will be disseminated through a peer-reviewed article.

\section{Consent for publication}

Not applicable.

\section{Conflict of interest}

None

Funding

No

\section{Authors' contributions}

Linda Zhong and ZB were responsible for the conception and design of this study. LWC and LY performed the search and evaluated studies for inclusion. LWC and JC extracted data from selected RCTs. JC and SL assessed the quality of selected RCTs .CSY and BFL Ng assessed the quality of certainty of the evidence. HJL and ECL Yu performed data analysis. ZB and AYL reviewed the work. WCL and HJL drafted the paper. All authors critically revised and approved the final paper.

\section{References}

1. World Health Organization. WHO reveals leading causes of death and disability worldwide: 2000-2019. 2020. Available from: https://www.who.int/news/item/09-12-2020-who-reveals-leading-causes-of-death-and-disability-worldwide-2000-2019.

2. Fridriksson J, Hillis AE. Current Approaches to the Treatment of Post-Stroke Aphasia. J Stroke. 2021;23(2):183-201.

3. Kim CY, Kim HD. Effect of crossed-education using a tilt table task-oriented approach in subjects with post-stroke hemiplegia: A randomized controlled trial. J Rehabil Med. 2018;50(9):792-799.

4. Fang J, Tuo M, Ouyang K, Xu Y. Statin on post-stroke epilepsy: a systematic review and meta-analysis. J Clin Neurosci. 2021;83:83-87.

5. Weaver NA, Kuijf HJ, Aben HP, et al. Strategic infarct locations for post-stroke cognitive impairment: a pooled analysis of individual patient data from 12 acute ischaemic stroke cohorts. Lancet Neurol. 2021;20(6):448-459.

6. Cai W, Mueller C, Li YJ, Shen WD, Stewart R. Post stroke depression and risk of stroke recurrence and mortality: a systematic review and meta-analysis. Ageing Res Rev. 2019;50:102-109. 
7. Das J, G KR. Post stroke depression: The sequelae of cerebral stroke. Neurosci Biobehav Rev. 2018;90:104-114.

8. Ahmed ZM, Khalil MF, Kohail AM, Eldesouky IF, Elkady A, Shuaib A. The Prevalence and Predictors of Post-Stroke Depression and Anxiety During COVID-19 Pandemic. J Stroke Cerebrovasc Dis. 2020;29(12):105315.

9. Schottke H, Giabbiconi CM. Post-stroke depression and post-stroke anxiety: prevalence and predictors. Int Psychogeriatr. 2015;27(11):18051812.

10. Oni OD, Olagunju AT, Olisah VO, Aina OF, Ojini FI. Post-stroke depression: Prevalence, associated factors and impact on quality of life among outpatients in a Nigerian hospital. S Afr J Psychiatr. 2018;24:1058-1058.

11. Villa RF, Ferrari F, Moretti A. Post-stroke depression: Mechanisms and pharmacological treatment. Pharmacol Ther. 2018;184:131-144.

12. Hackam DG, Mrkobrada M. Selective serotonin reuptake inhibitors and brain hemorrhage: a meta-analysis. Neurology. 2012;79(18):18621865.

13. Lee YC, Lin CH, Lin MS, Lin JW, Chang CH, Lai MS. Effects of selective serotonin reuptake inhibitors versus tricyclic antidepressants on cerebrovascular events: a nationwide population-based cohort study. J Clin Psychopharmacol. 2013;33(6):782-789.

14. Lu L, Zhang XG, Zhong LL, et al. Acupuncture for neurogenesis in experimental ischemic stroke: a systematic review and meta-analysis. Sci Rep. 2016;6:19521.

15. Vickers AJ, Vertosick EA, Lewith G, et al. Acupuncture for Chronic Pain: Update of an Individual Patient Data Meta-Analysis. $J$ Pain. 2018;19(5):455-474.

16. Zhang Y, Lin L, Li H, Hu Y, Tian L. Effects of acupuncture on cancer-related fatigue: a meta-analysis. Supportive care in cancer. 2018;26(2):415-425.

17. Zhang XY, Li YX, Liu DL, Zhang BY, Chen DM. The effectiveness of acupuncture therapy in patients with post-stroke depression: An updated meta-analysis of randomized controlled trials. Medicine (Baltimore). 2019;98(22):e15894.

18. Wang X, Xiong J, Yang J, et al. Meta-analysis of the clinical effectiveness of combined acupuncture and Western Medicine to treat poststroke depression. J Tradit Chin Med. 2021;41(1):6-16.

19. Zhang L, Zhao J, Quan S-I, et al. Effect of acupuncture plus Tai Ji Quan on the recovery of neurological function and depression state in post-stroke depression patients. Journal of Acupuncture and Tuina Science. 2018;16(2):96-103.

20. Yao L-j, Zhaoqing L, Jin Z, Keli W. Clinical study of tongdu Tiaoshen Acupuncture combined with cognitive therapy on intervention of poststroke depression patients (Chinese). World Science and Technology-Modernization of Traditional Chinese medicine. 2017;19(08):13191323.

21. Shim SR, Kim SJ, Lee J, Rücker G. Network meta-analysis: application and practice using R software. (2092-7193 (Electronic)).

22. Hutton B SG, Caldwell DM, Chaimani A, Schmid CH, Cameron C, loannidis JP, Straus S, Thorlund K, Jansen JP, Mulrow C, Catalá-López F, Gøtzsche PC, Dickersin K, Boutron I, Altman DG, Moher D. The PRISMA Extension Statement for Reporting of Systematic Reviews Incorporating Network Meta-analyses of Health Care Interventions: Checklist and Explanations. Ann Intern Med. 2015;162(11):777-784.

23. Wai Ching LAM CSY, Peijing Yan, Hin Yu Jeanie Cheung, Lidan Zhong. Acupuncture treatments for post-stroke rehabilitation: a systematic review and network meta-analysis. PROSPERO 2020 CRD42020218752 Available from: https://www.crd.york.ac.uk/prospero/display_record.php?ID=CRD42020218752.

24. Sterne JAC SJ, Page MJ, Elbers RG, Blencowe NS, Boutron I, Cates CJ, Cheng H-Y, Corbett MS, Eldridge SM, Hernán MA, Hopewell S, Hróbjartsson A, Junqueira DR, Jüni P, Kirkham JJ, Lasserson T, Li T, McAleenan A, Reeves BC, Shepperd S, Shrier I, Stewart LA, Tilling K, White IR, Whiting PF, Higgins JPT. RoB 2: a revised tool for assessing risk of bias in randomised trials. BMJ $2019 ; 366$ : I4898.

25. MacPherson H, Altman DG, Hammerschlag R, et al. Revised STandards for Reporting Interventions in Clinical Trials of Acupuncture (STRICTA): extending the CONSORT statement. PLoS Medicine. 2010;7(6):e1000261.

26. Citation: Nikolakopoulou A HJ, Papakonstantinou T, Chaimani A, Del Giovane C, Egger M, et al. (2020) CINeMA: An approach for assessing confidence in the results of a network meta-analysis. PLoS Med 17(4): e1003082. https://doi.org/10.1371/journal.pmed.1003082.

27. Guyatt G, Oxman Ad Fau - Akl EA, Akl Ea Fau - Kunz R, et al. GRADE guidelines: 1. Introduction-GRADE evidence profiles and summary of findings tables. (1878-5921 (Electronic)).

28. Xiao C. Clinical study of combined acupuncture and medicine in the treatment of depression after stroke. Chinese Community Doctors. 2012;14(11):237-238.

29. Chen A-w, Yuan G, Guantao W, LI J, Shen W-d. Effects of early acupuncture intervention on post-stroke depression: a randomized controlled study (Chinese). Chinese acupuncture and moxibustion. 2018;38(11):1141-1144.

30. Loujie C, Fangyuan S, Yaqi H, Dandan L. Effect of wake - up and Open - closing acupuncture on post-stroke depression and its influence on serum 5-HT content (Chinese). GUIDING JOURNAL OF TCM. 2018;24(14):38-40+43.

31. Cheng Y, Qisheng T. A randomized controlled observation of abdominal acupuncture in the treatment of depression syndrome of Liver depression and spleen deficiency syndrome after cerebral apoplexy in the elderly (Chinese). Chinese Tissue Engineering Research and

Page 7/16 
Clinical Rehabilitation. 2007(39):7791-7794.

32. Chen $\mathrm{Y}$, Zhao J-j. A randomized controlled clinical study of abdominal acupuncture in the treatment of post-stroke depression (Chinese). chinese archives of traditional chinese medicine. 2007(09):1888-1891.

33. Shuqing D, Xiuyuan S, Xaioyu L. Clinical observation on treatment of post-stroke depression with Bupleurum plus Longguoyasu decoction and acupuncture (Chinese). Chinese journal of Integrated Traditional and Western Medicine Cardio-cerebrovascular diseases. 2010;8(09):1054-1055.

34. Huiqin D, Handong D, Jingjuan Y. Clinical study of electroacupuncture through point and body acupuncture combined with fluoxetine in the treatment of post-stroke depression (Chinese). Journal of New Chinese Medicine. 2020;52(03):143-146.

35. Gao W. Clinical study of acupuncture and moxibustion combined with cupping therapy for depression after stroke (Chinese). Electronic journal of Clinical Medicine literature. 2017;4(87):17088.

36. Aisong G, Ai-hong L, Xin C, Junyan C, Qinfeng W. Effect of electroacupuncture combined with fluoxetine on depressive state and neurological function in patients with post-stroke depression (Chinese). Shandong Medical Journal. 2011;51(25):9-11.

37. guo R-y, SUN L, Liu L-a, Wang C-x. ffect of Linggui Eight methods on curative effect and quality of life of patients with post-stroke depression (Chinese). Chinese acupuncture and moxibustion 2009;29(10):785-790.

38. Xijun H, Jilin T, Benguo W, Yingying L. Clinical observation of 86 cases of post-stroke depression treated by Xingnao Kaiqiao acupuncture (Chinese). ChinJRehabil Theory Pract. 2005(06):467-468.

39. Jun H, Shen P-f. Clinical effect of acupuncture on post-stroke depression (Chinese). Acupuncture Research. 2007(01):58-61.

40. HUANG L. 30 cases of post-stroke depression treated by TCM combined with acupuncture (Chinese). Journal of emergency in traditional chinese medicine. 2011;20(05):802-803.

41. HUANG S-I, Wei Y, Zhang Z. Clinical randomized controlled study of electroacupuncture "Shenwuxing Group point" combined with Western medicine in the treatment of post-stroke depression (Chinese). Shanghai Journal of Traditional Chinese Medicine. 2014;48(07):33-36.

42. Lei J, Jun z. Clinical study of acupuncture and moxibustion combined with drugs in the treatment of post-stroke depression (Chinese). Journal of clinical acupuncture and moxibustion. 2011;27(11):15-17.

43. Jiang Z, Gao L, Li C, Li J. Clinical Study of Electroacupuncture at "Niwan Bazhen" Point in the Treatment of Depression after Stroke (Chinese). World Journal of Acupuncture-Moxibustion. 2007(01):11-16.

44. Jiao H-f, Liu W-m, Hao X, Zhang L. Clinical observation of electroacupuncture and paroxetine in the treatment of post-stroke depression in 33 cases (Chinese). Medical Review. 2012;18(06):950-951.

45. li L, Jiali J. Clinical observation of electroacupuncture combined with paroxetine in the treatment of post-stroke depression (Chinese). Journal of gansu college of traditional chinese medicine. 2011;28(01):53-54.

46. Ziling L, Jun Z, Fanqiang L. Effect of acupuncture on rehabilitation of post-stroke depression (Chinese). China Practical Medical. 2010;5(08):59-60.

47. Liu S, ZHAO S, Xi Z. Incidence of post-stroke depression and acupuncture treatment (Chinese). Chinese acupuncture and moxibustion. 2006(07):472-474.

48. Yanxiang L, Liping C, Ziqi Z, et al. Influence of "Wisdom three acupuncture" and syndrome differentiation on the curative effect of poststroke depression (Chinese). GUIDING JOURNAL OF TCM. 2010;16(05):116-117.

49. NIE R, HUANG C. Efficacy and safety of acupuncture in the treatment of post-stroke depression (Chinese). Chinese acupuncture and moxibustion. 2013;33(06):490-494.

50. Nie R, HUANG C, FU W-b. Observation on the effect of treating post-stroke depression from spleen and stomach (Chinese). Chinese acupuncture and moxibustion. 2011;31(04):325-328.

51. Peng H, Ye J, He X, Tan J, Zhang Z. Clinical randomized controlled study of electroacupuncture and fluoxetine capsule in the treatment of post-stroke depression (Chinese). JILIN journal of traditional chinese medicine. 2011;31(10):990-992.

52. Shuchang S, Zhi L, Runyun W, Hua C. Effect of acupuncture on cerebral apoplexy depression and neurological rehabilitation (Chinese). Journal of clinical acupuncture and moxibustion. 2014;30(10):7-9.

53. Su Z-w, Zhang Z-w. Thirty cases of post-stroke depression treated by pricking blood and channeling Meridian (Chinese). Shaanxi J Tradit Chin Med. 2010;31(12):1651-1652.

54. RuHua S, lei Z, RuBo S. Clinical observation of 72 cases of acute stroke depression treated by acupuncture (Chinese). Chinese ethnic and folk medicine. 2009;18(11):94-95.

55. Peiyang S, Haoran C, LI P-f, et al. Effect of Tongdu Tiaoshen acupuncture on drug treatment of post-stroke depression (Chinese). Chinese acupuncture and moxibustion. 2015;35(08):753-757.

56. Peiyang S, Haoran C, Li P-f, et al. The treatment of post-stroke depression with acupuncture therapy: a randomized controlled study (Chinese). Chinese acupuncture and moxibustion. 2013;33(01):3-7.

Page $8 / 16$ 
57. Wenge S, Shukai H, Cao W-j, et al. Effect of tonifying Qi and resolving phlegm and collaterals prescription combined with external and internal meridian acupuncture on depressive state of patients with shoulder and hand syndrome after stroke (Chinese). Chinese Medicine Guide. 2012;18(03):16-18.

58. Tong X, Liu D-d, Wei Y, Kou J, Yang T, Qiao L. Intervention effect of Regulating spirit and regulating liver acupuncture on post-stroke depression (Chinese). China Medicine and Pharmacy. 2012;2(03):127-128.

59. Laiqun W. Analysis of application of acupuncture and medicine in treating apoplexy sequelae (Chinese). Chinese Traditional Medicine modern distance education. 2010;8(11):216-217.

60. wang Y-h, Shuqin H, Lijing C, Baoli Z, Jun X. Effects of early rehabilitation training combined with abdominal therapy on motor function and mental disorders in stroke patients (Chinese). Chinese acupuncture and moxibustion. 2016;36(06):577-580.

61. Wang Y, Zhou T, Xiang S, Yonglei Z, Fang W. Observation on curative effect of Jieyu Qingxin Decoction combined with Tongdu Tiaoshen Acupuncture on post-stroke depression (Chinese). Traditional Chinese Medicine in Sichuan Province. 2020;38(08):135-139.

62. WU Y, sun X-h, XING Y. Intervention effect of penetrating acupuncture on post-stroke depression and influence on plasma cortisol level (Chinese). Shanghai Journal of Acupuncture and Moxibustion. 2011;30(03):153-154.

63. Jiaping W. Clinical observation on 150 cases of post-apoplexy depression treated by acupuncture syndrome differentiation (Chinese). Acupuncture Research. 2010;35(04):303-306.

64. Siqi W, Ouyang B. Clinical observation on treatment of post-stroke depression with liver and kidney deficiency by penetrating acupuncture (Chinese). Journal of Shanxi college of traditional chinese medicine. 2020;36(05):39-41.

65. Xiaoling W. Effect of oral Prozac plus acupuncture on depression after stroke (Chinese). Modern Doctor of China. 2009;47(27):87-88.

66. Wei X, Hongbing K, Zhen W, et al. Clinical study of xiang Cong acupuncture combined with fluoxetine in the treatment of post-stroke depression (Chinese). Clinical Journal of Traditional Chinese Medicine. 2009;21(04):330-331.

67. XU G. A randomized parallel control study of acupuncture combined with fluoxetine in the treatment of depression after stroke (Chinese). Journal of practical traditional chinese internal medicine. 2014;28(11):158-160.

68. Changchang Y, Shaoyong S, Genka T, Zhanli Y. Effect of acupuncture on post-stroke depression (Chinese). Shaanxi J Tradit Chin Med. 2018;39(01):122-124.

69. YUE L. Observation on the curative effect of head acupuncture combined with auricular point sticking in the treatment of post-stroke depression (Chinese). Shanghai Journal of Acupuncture and Moxibustion. 2016;35(07):796-798.

70. Lin Z, Yan Z, QUAN S, et al. Acupuncture combined with auricular point pressing in the treatment of post-stroke depression: a randomized controlled study (Chinese). Chinese acupuncture and moxibustion. 2017;37(06):581-585.

71. Zhang R, WANG X. Treatment of 39 cases of depression after cerebral apoplexy by tiaoshen Tongluo acupuncture combined with repeated transcranial magnetic stimulation (Chinese). Shaanxi J Tradit Chin Med. 2011;32(01):86-87+94.

72. Zahng W, Shaolu C, Lin A-j, et al. Effects of combination of acupuncture and medicine on depressive state and daily living ability of patients with post-stroke depression (Chinese). Shanghai Journal of Acupuncture and Moxibustion. 2011;30(12):823-825.

73. Zhien Z, Ping L, Xiangwen X, Weifu D. A multicenter randomized parallel control study of Anshen Jieyu Decoction combined with acupuncture at Baihui Point in the treatment of post-stroke depression (Chinese). Shaanxi J Tradit Chin Med. 2020;41(03):390-393.

74. Zhu Y-g, Qin Z, to BX, su Q-I. Treatment of post-stroke depression by cephalic acupuncture combined with auricular sticking (Chinese). Journal of clinical acupuncture and moxibustion. 2012;28(02):17-18.

75. FengKui Z, Jun Z, Yong D. Clinical observation of acupuncture combined with medicine in the treatment of depression after stroke. Journal of clinical acupuncture and moxibustion. 2010;26(05):10-11.

76. Zhang L, Zhao J, Quan SL, Liu YH, Zhong Y. Effect of acupuncture plus Tai Ji Quan on the recovery of neurological function and depression state in post-stroke depression patients. Journal of Acupuncture and Tuina Science. 2018;16(2):96-103.

77. Xiaojing D, Junming F. Clinical observation on acupuncture treatment of depression after stroke. Medical Innovation in China. 2012;09(003):107-107.

78. Guomin J. Observation on the therapeutic effect of psychological counseling combined with electroacupuncture on post-stroke depression (Chinese). Chinese Journal of Modern Nursing Modern Nursing. 2007(35).

79. LI H, ZHONG B, FAN Y, HU H. Acupuncture for post-stroke depression: a randomized controlled study (Chinese). Chinese acupuncture and moxibustion. 2011;031(001):3-6.

80. Aibing Z, tan L. Clinical study of combined acupuncture and medicine in the treatment of post-stroke depression (Chinese). World Health Digest. 2009;6(8):59-60.

81. Guibo Z, ling Y, Qian Z. Effect of acupuncture on depression after stroke. Shanghai Journal of Acupuncture and Moxibustion. 2010(10):636637. 
82. Zhongjin Z, Jianhua C, Guifen X. Effect of early acupuncture combined with rehabilitation training on rehabilitation of depression after cerebral infarction (Chinese). Massage and rehabilitation medicine. 2011;02(006):54-55.

83. Zhao X-B. Effect of acupuncture on post-stroke depression. China Healthcare Innovation. 2012;07(006):38-39.

84. Yafen Z, Louni C, chao W, fang C, fang J-q. Single center randomized controlled trial of acupuncture for post-stroke depression. Journal of Zhejiang Traditional Chinese Medicine University. 2014(11):1322-1325.

85. Zhu J. Effect analysis of acupuncture and moxibustion combined with drugs in the treatment of post-stroke depression. Massage and rehabilitation medicine. 2012;3(011):372-372.

86. ZHAO H. One hundred patients with post-stroke depression treated by Xingnao Kaiqiao acupuncture were randomized controlled by stratification (Chinese). Chin J Clin Rehabil. 2003(31):117.

87. Zhao X. Effect of acupuncture on post-stroke depression(Chinese). China Healthcare Innovation. 2012;07(006):38-39.

88. Yan C, Su S, Tang G, Yu Z. Clinical study on acupuncture and moxibustion in the treatment of post-stroke depression(Chinese). Shanxi J Tradit Chin Med. 2018;v.39;No.433(01):124-126.

89. Klomjai W, Katz R, Lackmy-Vallée A. Basic principles of transcranial magnetic stimulation (TMS) and repetitive TMS (rTMS). Annals of Physical and Rehabilitation Medicine. 2015;58(4):208-213.

90. Lefaucheur JP, Aleman A, Baeken C, et al. Evidence-based guidelines on the therapeutic use of repetitive transcranial magnetic stimulation (rTMS): An update (2014-2018). Clin Neurophysiol. 2020;131(2):474-528.

91. Hang X, Li J, Zhang Y, et al. Efficacy of frequently-used acupuncture methods for specific parts and conventional pharmaceutical interventions in treating post-stroke depression patients: A network meta-analysis. Complementary Therapies in Clinical Practice. 2021;45:101471.

\section{Tables}

Table 1. Description of included studies. 


\begin{tabular}{|c|c|c|c|c|c|c|c|}
\hline \multirow[t]{2}{*}{ Study } & \multirow{2}{*}{$\begin{array}{l}\text { Age } \\
\text { (Treatment /Control) }\end{array}$} & \multirow[t]{2}{*}{ Arm } & \multirow{2}{*}{$\begin{array}{l}\text { Sample } \\
\text { size } \\
\text { (Treatment } \\
\text { /Control) }\end{array}$} & \multicolumn{3}{|c|}{ Interventions } & \multirow{2}{*}{$\begin{array}{l}\text { Follow } \\
\text { up }\end{array}$} \\
\hline & & & & Group 1 & Group 2 & Group 3 & \\
\hline $\begin{array}{l}\text { Xin TONG } \\
2012^{58}\end{array}$ & $58.5 \pm 8.4$ & 2 & $30 / 30$ & $A C+W M$ & WM & - & $8 w$ \\
\hline $\begin{array}{l}\text { Yanxiang } \\
\text { LIU } 2010^{48}\end{array}$ & male $63.8 \pm 10.0 /$ female $59.8 \pm 8.7$ & 2 & $40 / 40$ & $\mathrm{AC}$ & UC & - & $4 w$ \\
\hline $\begin{array}{l}\text { Siqi WU } \\
2020^{64}\end{array}$ & $60.1 \pm 10.7 / 58.3 \pm 11.2$ & 2 & $30 / 30$ & $A C+W M$ & WM & - & $8 w$ \\
\hline $\begin{array}{l}\text { Xiaoling Wu } \\
2009^{65}\end{array}$ & $56 \pm 9.63$ & 2 & $38 / 36$ & $A C+W M$ & WM & - & $40 d$ \\
\hline $\begin{array}{l}\text { Lijun YAO } \\
2017^{20}\end{array}$ & $65 \pm 11 / 63 \pm 12$ & 2 & $30 / 30$ & $\mathrm{AC}+\mathrm{CT}$ & WM & - & $4 w$ \\
\hline $\begin{array}{l}\text { Lei JIANG } \\
2011^{42}\end{array}$ & $58.93 \pm 3.58 / 59.67 \pm 3.43$ & 2 & $30 / 30$ & $A M+W M$ & WM & - & $6 w$ \\
\hline $\begin{array}{l}\text { Peiyang } \\
\text { SUN } 2013^{56}\end{array}$ & $58 \pm 8 / 59 \pm 9$ & 2 & $30 / 30$ & $\mathrm{AC}$ & WM & - & $4 w$ \\
\hline $\begin{array}{l}\text { Peiyang } \\
\text { SUN } 2015^{55}\end{array}$ & $59 \pm 7 / 58 \pm 8$ & 2 & $33 / 30$ & $\mathrm{AC}$ & WM & - & $4 w$ \\
\hline $\begin{array}{l}\text { Xiao CHANG } \\
2012^{28}\end{array}$ & $56.8 / 55.9$ & 2 & $32 / 30$ & $\mathrm{AC}+\mathrm{TCM}$ & WM & - & $6 w$ \\
\hline $\begin{array}{l}\text { Zhongjin } \\
\text { ZHANG } \\
2011^{82}\end{array}$ & $56.7 \pm 12.1 / 55.8 \pm 9.7$ & 2 & $40 / 38$ & $\mathrm{AC}$ & UC & - & $3 w$ \\
\hline $\begin{array}{l}\text { Guibo } \\
\text { ZHANG } \\
2010^{81}\end{array}$ & $65 \pm 5 / 66 \pm 5$ & 2 & $30 / 30$ & $A C+W M$ & WM & - & $4 w$ \\
\hline $\begin{array}{l}\text { Ru ZHANG } \\
2011^{71}\end{array}$ & 50.1-71.2/49.9-68.7 & 2 & $39 / 39$ & AC+RTMS & AC & - & $4 w$ \\
\hline $\begin{array}{l}\text { Lin ZHANG } \\
2017^{70}\end{array}$ & $59 \pm 9 / 58 \pm 8$ & 2 & $30 / 30$ & $\mathrm{AC}$ & WM & - & $8 w$ \\
\hline $\begin{array}{l}\text { Gang XU } \\
2014^{67}\end{array}$ & $59.4 \pm 7.6 / 60.8 \pm 6.9$ & 2 & $20 / 18$ & $A C+W M$ & WM & - & $6 w$ \\
\hline $\begin{array}{l}\text { Shuqing DAI } \\
2010^{33}\end{array}$ & $64.36 \pm 5.46 / 65.32 \pm 5.09$ & 2 & $24 / 24$ & $\mathrm{AC}+\mathrm{TCM}$ & WM & - & $4 w$ \\
\hline $\begin{array}{l}\text { Jian ZHU } \\
2012^{85}\end{array}$ & NR & 2 & $20 / 20$ & $A M+W M$ & WM & - & $6 w$ \\
\hline $\begin{array}{l}\text { Yonggang } \\
\text { ZHU } 2012^{74}\end{array}$ & $67.3 \pm 4.5 / 68.9 \pm 6.1$ & 2 & $21 / 21$ & AC & WM & - & $8 w$ \\
\hline Li LI $2011^{45}$ & $65 \pm 13 / 63 \pm 12$ & 2 & $20 / 19$ & AC & WM & - & $4 w$ \\
\hline $\begin{array}{l}\text { Hongjie LI } \\
2011^{79}\end{array}$ & $29-60 / 32-63$ & 2 & $23 / 20$ & AC & WM & - & $6 w$ \\
\hline $\begin{array}{l}\text { Ziling LIN } \\
2010^{46}\end{array}$ & $52-84$ & 2 & $20 / 20$ & AC & UC & - & $4 w$ \\
\hline $\begin{array}{l}\text { Yun WU } \\
2011^{62}\end{array}$ & $62 \pm 11 / 64 \pm 11$ & 2 & $36 / 36$ & $A C$ & WM & - & $8 w$ \\
\hline $\begin{array}{l}\text { Haifeng } \\
\text { JIAO } 2012^{44}\end{array}$ & $43-78,63.3 / 45-76$ & 2 & $17 / 16$ & $A C+W M$ & WM & - & $4 w$ \\
\hline
\end{tabular}




\begin{tabular}{|c|c|c|c|c|c|c|c|}
\hline $\begin{array}{l}\text { Laiqun } \\
\text { WANG } \\
2010^{59}\end{array}$ & $40-70 / 40-68$ & 2 & $30 / 30$ & $\mathrm{AC}+\mathrm{TCM}$ & WM & - & $8 w$ \\
\hline $\begin{array}{l}\text { Changchang } \\
\text { YAN } 2018^{88}\end{array}$ & $62.1 \pm 13.5 / 62.3 \pm 13.6$ & 2 & $45 / 45$ & $A M+W M$ & WM & - & $4 w$ \\
\hline $\begin{array}{l}\text { Yuan } \\
\text { CHENG } \\
2007^{31}\end{array}$ & $72 \pm 8 / 69 \pm 7 / 69 \pm 6$ & 3 & $19 / 20 / 21$ & special AC & $\mathrm{AC}$ & UC & $6 w$ \\
\hline $\begin{array}{l}\text { Yuan } \\
\text { CHENG } \\
2008^{32}\end{array}$ & $61.7 \pm 8.1 / 63.2 \pm 7.9 / 62.9 \pm 7.3$ & 3 & $20 / 20 / 20$ & special AC & $\mathrm{AC}$ & UC & $6 w$ \\
\hline $\begin{array}{l}\text { Rongrong } \\
\text { NIE } 2011^{50}\end{array}$ & $64.2 \pm 9.85 / 63.1 \pm 9.55$ & 2 & $33 / 30$ & $A C$ & WM & - & $4 w$ \\
\hline $\begin{array}{l}\text { Zhiwei SU } \\
2010^{53}\end{array}$ & $55.8 \pm 6.1 / 54.2 \pm 6.5$ & 2 & $30 / 30$ & $A C$ & WM & - & $30 d$ \\
\hline $\begin{array}{l}\text { Guomin } \\
\text { JIANG } \\
2007^{78}\end{array}$ & 60.5 & 2 & $25 / 25$ & AC+Psych & Psych & - & $4 w$ \\
\hline $\begin{array}{l}\text { Zhenya } \\
\text { JIANG } \\
2011^{43}\end{array}$ & $60.32 \pm 3.26 / 61.18 \pm 2.94$ & 2 & $33 / 33$ & $A C+W M$ & WM & - & $4 w$ \\
\hline $\begin{array}{l}\text { Xiaobing } \\
\text { ZHAO } \\
2012^{87}\end{array}$ & $56 \pm 13.9 / 57 \pm 12.8$ & 2 & $45 / 45$ & $A C+W M$ & WM & - & $20 d$ \\
\hline $\begin{array}{l}\text { Aisong GUO } \\
2011^{36}\end{array}$ & $40-65$ & 3 & $32 / 31 / 32$ & $A C$ & WM & $A C+W M$ & $6 w$ \\
\hline $\begin{array}{l}\text { Aiwen CHEN } \\
2017^{29}\end{array}$ & $57 \pm 11 / 58 \pm 11$ & 2 & $30 / 30$ & $A C$ & UC & - & $4 w$ \\
\hline $\begin{array}{l}\text { Lujie CHEN } \\
2018^{30}\end{array}$ & $62.37 \pm 6.28 / 60.21 \pm 5.78$ & 2 & $30 / 30$ & $\mathrm{AC}$ & WM & - & $4 w$ \\
\hline $\begin{array}{l}\text { Ruhua SUI } \\
2009^{54}\end{array}$ & $62.93 \pm 7.25$ & 2 & $36 / 36$ & $\mathrm{AC}$ & UC & - & $4 w$ \\
\hline $\begin{array}{l}\text { Wa GAO } \\
2017^{35}\end{array}$ & $58.12 \pm 3.27$ & 2 & $33 / 33$ & $A C$ & AC+Cupping+Psych & - & $10 d$ \\
\hline $\begin{array}{l}\text { Shile } \\
\text { HUANG } \\
2014^{41}\end{array}$ & $61.43 \pm 9.91 / 62.10 \pm 8.11 / 62.77 \pm 9.32$ & 3 & $30 / 30 / 30$ & $A C$ & WM & $A C+W M$ & $6 w$ \\
\hline $\begin{array}{l}\text { Long } \\
\text { HUANG } \\
2011^{40}\end{array}$ & $65.22 \pm 8.69 / 65.39 \pm 11.26 / 63.25 \pm 10.28$ & 3 & $30 / 30 / 30$ & $\mathrm{AC}+\mathrm{TCM}$ & $\mathrm{TCM}$ & WM & $60 d$ \\
\hline $\begin{array}{l}\text { Aibing } \\
\text { ZHANG } \\
2009^{80}\end{array}$ & $63 \pm 11$ total & 2 & $40 / 35$ & $\mathrm{AM}+\mathrm{TCM}$ & WM & - & $30 d$ \\
\hline $\begin{array}{l}\text { Xiaojing } \\
\text { DUAN } \\
2012^{77}\end{array}$ & $58.80 \pm 9.60 / 60.22 \pm 8.12$ & 2 & $30 / 30$ & $\mathrm{AC}$ & WM & - & $8 w$ \\
\hline $\begin{array}{l}\text { Fengkui } \\
\text { ZHU } 2010^{75}\end{array}$ & NR & 2 & $30 / 30$ & $A C+W M$ & WM & - & $4 w$ \\
\hline $\begin{array}{l}\text { Yahui WANG } \\
2016^{60}\end{array}$ & $66 \pm 6 / 65 \pm 7 / 66 \pm 8$ & 3 & $30 / 30 / 30$ & $A C$ & UC & - & $4 w$ \\
\hline $\begin{array}{l}\text { Wenge SUN } \\
2012^{57}\end{array}$ & $54.7 \pm 2.9 / 54.2 \pm 3.1$ & 2 & $50 / 40$ & $\mathrm{AC}+\mathrm{TCM}$ & UC & - & $3 w$ \\
\hline Ruiyou GUO & $65.8 \pm 9.61 / 67.6 \pm 12.43 / 64.5 \pm 12.07$ & 3 & $40 / 40 / 40$ & $A C$ & WM & UC & $6 w$ \\
\hline
\end{tabular}

Page 12/16 


\begin{tabular}{|c|c|c|c|c|c|c|c|}
\hline $\begin{array}{l}\text { Sukun LIU } \\
2006^{47}\end{array}$ & $60.0 \pm 9.8 / 59.6 \pm 8.9$ & 2 & $101 / 145$ & $\mathrm{AC}$ & WM & - & $6 w$ \\
\hline $\begin{array}{l}\text { Yafen ZHOU } \\
2014^{84}\end{array}$ & $64 \pm 15 / 65 \pm 11$ & 2 & $75 / 72$ & $A C+W M$ & WM & - & $8 w$ \\
\hline $\begin{array}{l}\text { Huiyuan } \\
\text { PENG } \\
2011^{51}\end{array}$ & $64.6 \pm 11.3 / 73.7 \pm 11.8$ & 2 & $58 / 59$ & $\mathrm{AC}$ & WM & - & $4 w$ \\
\hline $\begin{array}{l}\text { Wei ZHANG } \\
2011^{72}\end{array}$ & $28-80 / 30-83 / 44-85$ & 3 & $52 / 49 / 49$ & $\mathrm{AC}+\mathrm{TCM}$ & $\mathrm{AC}$ & TCM & $45 d$ \\
\hline $\begin{array}{l}\text { Yaqun } \\
\text { WANG } \\
2020^{61}\end{array}$ & $70.44 \pm 5.62 / 70.53 \pm 6.44$ & 2 & $52 / 51$ & $\mathrm{AC}+\mathrm{TCM}+\mathrm{WM}$ & WM & - & $60 d$ \\
\hline $\begin{array}{l}\text { Rongrong } \\
\text { NIE } 2013^{49}\end{array}$ & $64 \pm 10 / 63 \pm 10 / 64 \pm 10$ & 3 & $42 / 41 / 40$ & $A M+W M$ & AM & WM & $4 w$ \\
\hline $\begin{array}{l}\text { Hong ZHAO } \\
2003^{86}\end{array}$ & NR & 2 & $50 / 50$ & $\mathrm{AC}$ & WM & - & $8 w$ \\
\hline $\begin{array}{l}\text { Wei XIAO } \\
2009^{66}\end{array}$ & $53 \pm 6 / 51 \pm 8$ & 2 & $40 / 40$ & $A C+W M$ & WM & - & $30 d$ \\
\hline $\begin{array}{l}\text { Huiqin DING } \\
2020^{34}\end{array}$ & $54.63 \pm 3.51 / 54.27 \pm 3.43$ & 2 & $61 / 61$ & $A C+W M$ & WM & - & $4 w$ \\
\hline $\begin{array}{l}\text { Jiaping WU } \\
2010^{63}\end{array}$ & $56.2 \pm 9.2 / 55.7 \pm 9.4$ & 2 & $150 / 150$ & $\mathrm{AC}$ & WM & - & $60 d$ \\
\hline $\begin{array}{l}\text { Shuchang } \\
\text { SONG } \\
2014^{52}\end{array}$ & $57.25 \pm 11.32 / 57.46 \pm 11.57$ & 2 & $100 / 100$ & $\mathrm{AC}$ & WM & - & $8 w$ \\
\hline $\begin{array}{l}\text { L. ZHANG } \\
2018^{76}\end{array}$ & $63.7 \pm 6.8 / 62.4 \pm 7.6$ & 2 & $45 / 45$ & AC+ Tai Chi & WM & - & $30 d$ \\
\hline $\begin{array}{l}\text { Zhien ZHOU } \\
2020^{73}\end{array}$ & $64.52 \pm 1.10 / 65.16 \pm 12.60$ & 2 & $140 / 85$ & $\mathrm{AC}+\mathrm{TCM}+\mathrm{WM}$ & WM & - & $12 d$ \\
\hline $\begin{array}{l}\text { Jun HE } \\
2007^{39}\end{array}$ & $53.16 \pm 11.20 / 53.86 \pm 12.34$ & 2 & $180 / 76$ & $\mathrm{AC}$ & WM & - & $30 d$ \\
\hline $\begin{array}{l}\text { Xijun HE } \\
2005^{38}\end{array}$ & $41-72 / 39-71$ & 2 & $86 / 86$ & $\mathrm{AC}$ & WM & - & $8 w$ \\
\hline
\end{tabular}

NOTE: AC, Acupuncture; AM, Acupuncture and moxibustion; TCM, Traditional Chinese medicine; WM, Western medicine; UC, Usual care; RTMS, Repetitive transcranial magnetic stimulation; CT, Cognitive therapy.

Table 2. The result of NMA for depression. 


\begin{tabular}{|c|c|c|c|c|c|c|c|c|c|c|c|}
\hline \multicolumn{12}{|c|}{ AC+RTMS } \\
\hline $\begin{array}{l}-2.93 \\
(-12.58 \\
6.7)\end{array}$ & $\mathrm{AC}+\mathrm{TCM}+\mathrm{WM}$ & & & & & & & & & & \\
\hline $\begin{array}{l}-2.08 \\
(-10.73 \\
6.59)\end{array}$ & $\begin{array}{l}0.85(-5.55 \\
7.28)\end{array}$ & $\mathrm{AC}+\mathrm{TCM}$ & & & & & & & & & \\
\hline $\begin{array}{l}-4.25 \\
(-15.38 \\
6.84)\end{array}$ & $\begin{array}{l}-1.33(-10.71, \\
8.09)\end{array}$ & $\begin{array}{l}-2.17 \\
(-10.66 \\
6.31)\end{array}$ & $\begin{array}{l}\mathrm{AC}+\mathrm{Tai} \\
\mathrm{Chi}\end{array}$ & & & & & & & & \\
\hline $\begin{array}{l}-4.79 \\
(-15.94 \\
6.36)\end{array}$ & $\begin{array}{l}-1.86(-11.3 \\
7.59)\end{array}$ & $\begin{array}{l}-2.72 \\
(-11.22 \\
5.77)\end{array}$ & $\begin{array}{l}-0.54 \\
(-11.51 \\
10.38)\end{array}$ & $\mathrm{AC}+\mathrm{CT}$ & & & & & & & \\
\hline $\begin{array}{l}-3.31 \\
(-12.82 \\
6.17)\end{array}$ & $\begin{array}{l}-0.39(-7.94 \\
7.17)\end{array}$ & $\begin{array}{l}-1.25 \\
(-6.49 \\
3.99)\end{array}$ & $\begin{array}{l}0.93 \\
(-8.42 \\
10.33)\end{array}$ & $\begin{array}{l}1.49 \\
(-7.92 \\
10.81)\end{array}$ & TCM & & & & & & \\
\hline $\begin{array}{l}-6.4 \\
(-16.96 \\
4.19)\end{array}$ & $\begin{array}{l}-3.48(-12.24, \\
5.34)\end{array}$ & $\begin{array}{l}-4.33 \\
(-12.07 \\
3.47)\end{array}$ & $\begin{array}{l}-2.15 \\
(-12.48 \\
8.26)\end{array}$ & $\begin{array}{l}-1.62 \\
(-11.98 \\
8.77)\end{array}$ & $\begin{array}{l}-3.07 \\
(-11.78 \\
5.67)\end{array}$ & $\mathrm{AM}$ & & & & & \\
\hline $\begin{array}{l}-4.4 \\
(-12.71 \\
3.86)\end{array}$ & $\begin{array}{l}-1.48(-7.32 \\
4.39)\end{array}$ & $\begin{array}{l}-2.34 \\
(-6.48 \\
1.84)\end{array}$ & $\begin{array}{l}-0.16 \\
(-8.19 \\
7.9)\end{array}$ & $\begin{array}{l}0.38 \\
(-7.68 \\
8.46)\end{array}$ & $\begin{array}{l}-1.08 \\
(-6.85 \\
4.67)\end{array}$ & $\begin{array}{l}1.99 \\
(-5.33 \\
9.28)\end{array}$ & $A C+W M$ & & & & \\
\hline $\begin{array}{l}-6.41 \\
(-15.29 \\
2.49)\end{array}$ & $\begin{array}{l}-3.47(-10.09 \\
3.19)\end{array}$ & $\begin{array}{l}-4.33 \\
(-9.52 \\
0.89)\end{array}$ & $\begin{array}{l}-2.16 \\
(-10.77 \\
6.47)\end{array}$ & $\begin{array}{l}-1.61 \\
(-10.26 \\
7.01)\end{array}$ & $\begin{array}{l}-3.07 \\
(-9.66 \\
3.49)\end{array}$ & $\begin{array}{l}0(-6.93 \\
6.92)\end{array}$ & $\begin{array}{l}-1.99 \\
(-6.48 \\
2.5)\end{array}$ & $A M+W M$ & & & \\
\hline $\begin{array}{l}-5.26 \\
(-13.07 \\
2.56)\end{array}$ & $\begin{array}{l}-2.33(-7.98 \\
3.36)\end{array}$ & $\begin{array}{l}-3.19 \\
(-6.92 \\
0.58)\end{array}$ & $\begin{array}{l}-1.01 \\
(-8.92 \\
6.88)\end{array}$ & $\begin{array}{l}-0.47 \\
(-8.39 \\
7.44)\end{array}$ & $\begin{array}{l}-1.93 \\
(-7.35 \\
3.45)\end{array}$ & $\begin{array}{l}1.14 \\
(-6.01 \\
8.27)\end{array}$ & $\begin{array}{l}-0.85 \\
(-3.6 \\
1.87)\end{array}$ & $\begin{array}{l}1.14 \\
(-3.08 \\
5.36)\end{array}$ & $\mathrm{AC}$ & & \\
\hline $\begin{array}{l}-8.75 \\
(-16.75 \\
-0.76)\end{array}$ & $\begin{array}{l}-5.83(-11.22, \\
-0.42)\end{array}$ & $\begin{array}{l}-6.68 \\
(-10.16 \\
-3.18)\end{array}$ & $\begin{array}{l}-4.5 \\
(-12.23 \\
3.2)\end{array}$ & $\begin{array}{l}-3.96 \\
(-11.7 \\
3.77)\end{array}$ & $\begin{array}{l}-5.43 \\
(-10.73 \\
-0.13)\end{array}$ & $\begin{array}{l}-2.35 \\
(-9.3 \\
4.58)\end{array}$ & $\begin{array}{l}-4.34 \\
(-6.64 \\
-2.05)\end{array}$ & $\begin{array}{l}-2.36 \\
(-6.21 \\
1.52)\end{array}$ & $\begin{array}{l}-3.49 \\
(-5.19 \\
-1.79)\end{array}$ & WM & \\
\hline $\begin{array}{l}-14.43 \\
(-22.81 \\
-6.08)\end{array}$ & $\begin{array}{l}-11.51(-17.85, \\
-5.17)\end{array}$ & $\begin{array}{l}-12.35 \\
(-17.11 \\
-7.62)\end{array}$ & $\begin{array}{l}-10.19 \\
(-18.59 \\
-1.83)\end{array}$ & $\begin{array}{l}-9.64 \\
(-18.1, \\
-1.21)\end{array}$ & $\begin{array}{l}-11.11 \\
(-17.28 \\
-4.99)\end{array}$ & $\begin{array}{l}-8.02 \\
(-15.73 \\
-0.34)\end{array}$ & $\begin{array}{l}-10.02 \\
(-14.04 \\
-6.04)\end{array}$ & $\begin{array}{l}-8.03 \\
(-13.17 \\
-2.94)\end{array}$ & $\begin{array}{l}-9.17 \\
(-12.17 \\
-6.22)\end{array}$ & $\begin{array}{l}-5.68 \\
(-9.04 \\
-2.33)\end{array}$ & UC \\
\hline
\end{tabular}

NOTE: The estimates of mean difference of treatments in the columns versus rows presented in the lower diagonal elements (while those of the row treatments vs. column treatments are presented in the upper diagonal elements). The MD of significant difference was presented in bold font. AC, Acupuncture; AM, Acupuncture and moxibustion; TCM, Traditional Chinese medicine; WM, Western medicine; UC, Usual care; RTMS, Repetitive transcranial magnetic stimulation; CT, Cognitive therapy.

Table 3. Ranking probability of different interventions. 


\begin{tabular}{|lll|}
\hline & Treatments & Cumulative Probability \\
\hline 1 & AC+RTMS & $49.43 \%$ \\
\hline 2 & AC+TCM+WM & $10.99 \%$ \\
\hline 3 & AC+TCM & $10.62 \%$ \\
\hline 4 & AC+Tai Chi & $10.30 \%$ \\
\hline 5 & AC+CT & $8.10 \%$ \\
\hline 6 & TCM & $7.48 \%$ \\
\hline 7 & AM & $2.53 \%$ \\
\hline 8 & AC+WM & $0.37 \%$ \\
\hline 9 & AM+WM & $0.16 \%$ \\
\hline 10 & AC & $0.01 \%$ \\
\hline 11 & WM & $0.00 \%$ \\
\hline 12 & UC & $0.00 \%$ \\
\hline
\end{tabular}

NOTE: AC, Acupuncture; AM, Acupuncture and moxibustion; TCM, Traditional Chinese medicine; WM, Western medicine; UC, Usual care; RTMS, Repetitive transcranial magnetic stimulation; CT, Cognitive therapy.

Table 4. The GRADE table for depression scores.

\begin{tabular}{|c|c|c|c|c|c|c|c|c|}
\hline Comparison & $\begin{array}{c}\text { Number } \\
\text { of } \\
\text { studies }\end{array}$ & Within-study bias & Reporting bias & Indirectness & Imprecision & Heterogeneity & Incoherence & Confidence rating \\
\hline$A C$ vs $A C$ with RTMS & 1 & Major concerns & Low risk & No concerns & Some concerns & Some concerns & No concerns & Low \\
\hline$A C$ vs $A C$ with TCM & 1 & No concerns & Low risk & No concerns & Some concerns & No concerns & No concerns & High \\
\hline$A C$ vs $A C$ with WM & 2 & Some concerns & Low risk & No concerns & No concerns & Major concerns & No concerns & Low \\
\hline AC vs TCM & 1 & No concerns & Low risk & No concerns & Some concerns & Some concerns & No concerns & Moderate \\
\hline AC vs UC & 7 & Some concerns & Low risk & No concerns & No concerns & Some concerns & No concerns & Moderate \\
\hline AC vs WM & 20 & Some concerns & Low risk & No concerns & No concerns & Some concerns & No concerns & Moderate \\
\hline AC with CT vs WM & 1 & Some concerns & Low risk & No concerns & Some concerns & Some concerns & No concerns & Moderate \\
\hline AC with TCM vs TCM & 2 & Major concerns & Low risk & No concerns & Some concerns & Some concerns & No concerns & Low \\
\hline AC with TCM vs WM & 4 & Major concerns & Low risk & No concerns & No concerns & Some concerns & No concerns & Low \\
\hline AC with TCM with WM vs WM & 2 & Some concerns & Low risk & No concerns & Some concerns & No concerns & No concerns & Moderate \\
\hline AC with Tai Chi vs WM & 1 & Major concerns & Low risk & No concerns & Some concerns & Some concerns & No concerns & Low \\
\hline AC with WM vs WM & 11 & Some concerns & Low risk & No concerns & No concerns & Some concerns & Some concerns & Moderate \\
\hline AM vs AM with WM & 1 & Some concerns & Low risk & No concerns & Major concerns & No concerns & No concerns & Low \\
\hline AM vs WM & 1 & Some concerns & Low risk & No concerns & Some concerns & Some concerns & No concerns & Moderate \\
\hline AM with WM vs WM & 4 & Some concerns & Low risk & No concerns & No concerns & Major concerns & No concerns & Low \\
\hline TCM vs WM & 1 & Major concerns & Low risk & No concerns & Some concerns & No concerns & No concerns & Low \\
\hline UC vs WM & 1 & Some concerns & Low risk & No concerns & No concerns & Some concerns & No concerns & Moderate \\
\hline
\end{tabular}

NOTE: AC, Acupuncture; AM, Acupuncture and moxibustion; TCM, Traditional Chinese medicine; WM, Western medicine; UC, Usual care; RTMS, Repetitive transcranial magnetic stimulation; CT, Cognitive therapy.

\section{Figures}




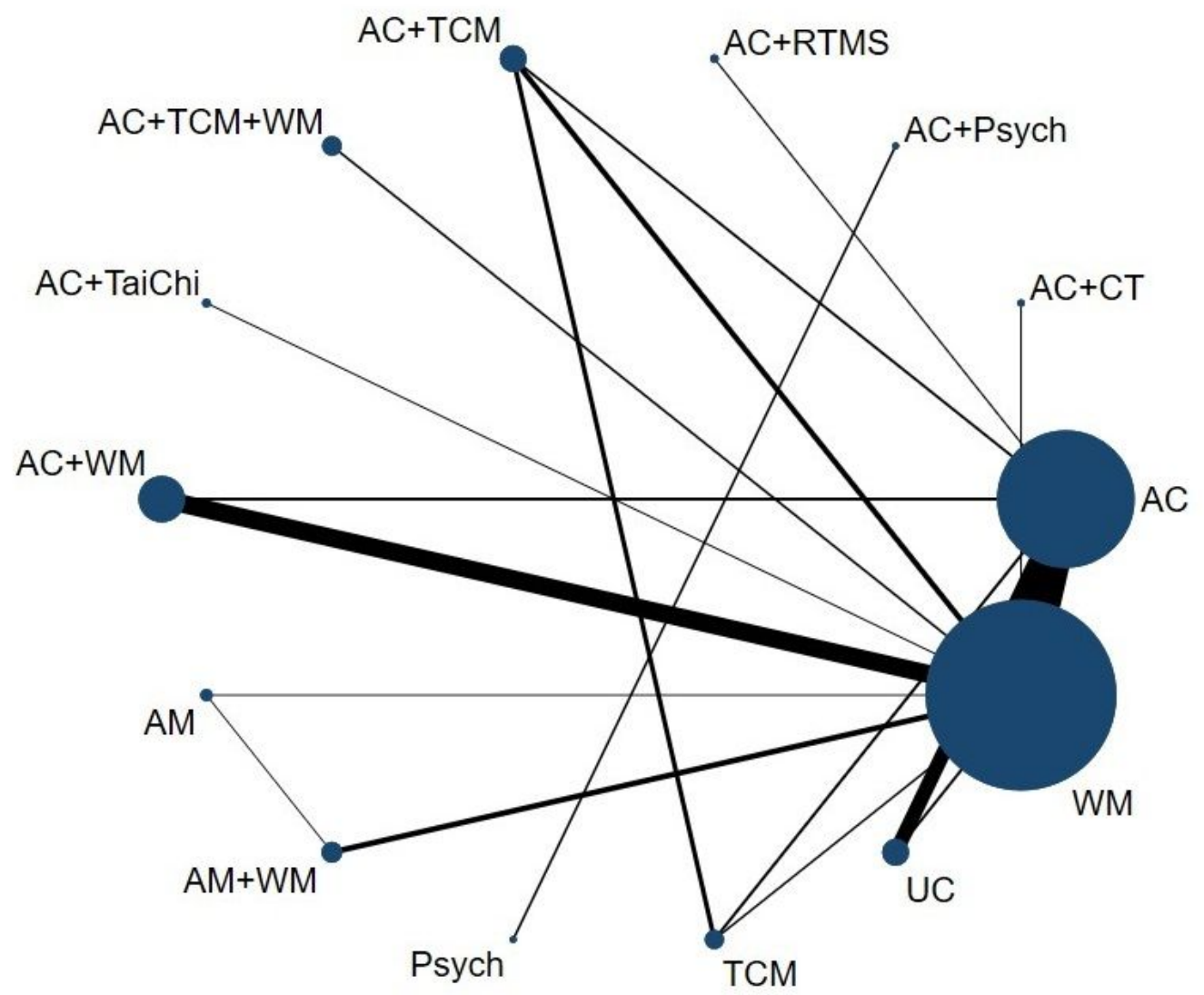

Figure 1

Network plot. NOTE: AC, Acupuncture; AM, Acupuncture and moxibustion; TCM, Traditional Chinese medicine; WM, Western medicine; UC, Usual care; RTMS, Repetitive transcranial magnetic stimulation; CT, Cognitive therapy.

\section{Supplementary Files}

This is a list of supplementary files associated with this preprint. Click to download.

- SupplementaryAppendix1.docx

- SupplementaryAppendix2.docx

- SupplementaryAppendix3.docx

- SupplementaryAppendix4.docx

- SupplementaryAppendix5.tif

- SupplementaryAppendix6.tif

- SupplementaryAppendix7.tif

- SupplementaryAppendix8.tif

- SupplementaryAppendix9.docx 